\title{
Global DNA methylation was changed by a maternal high-lipid, high-energy diet during gestation and lactation in male adult mice liver
}

\author{
Huan-Ling Yu†, Shan Dongt, Li-Fang Gao, Li Li, Yuan-Di Xi, Wei-Wei Ma, Lin-Hong Yuan and \\ Rong XiaO* \\ School of Public Health, Beijing Key Laboratory of Environmental Toxicology, Capital Medical University, Beijing 100069, \\ People's Republic of China
}

(Submitted 6 June 2014 - Final revision received 3 January 2015 - Accepted 13 January 2015 - First published online 17 March 2015)

\begin{abstract}
An epigenetic mechanism has been suggested to explain the effects of the maternal diet on the development of disease in offspring. The present study aimed to observe the effects of a maternal high-lipid, high-energy (HLE) diet on the DNA methylation pattern of male offspring in mice. Female C57BL/6J mice were fed an HLE diet during gestation and lactation. The genomic DNA methylations at promoter sites of genes in the liver, mRNA and protein levels of selected genes related to lipid and glucose metabolism were measured by microarray, real-time PCR and Western blot. The results indicated that the percentage of methylated DNA in offspring from dams that were fed an HLE diet was significantly higher than that from dams that were fed a chow diet, and most of these genes were hypermethylated in promoter regions. The nuclear protein content and mRNA levels of hypermethylated genes, such as PPAR $\gamma$ and liver X receptor $\alpha$ ( LXR $\alpha$ ), were decreased significantly in offspring in the HLE group. The results suggested that the DNA methylation profile in adult offspring livers was changed by the maternal HLE diet during gestation and lactation.
\end{abstract}

Key words: Maternal diet: Offspring: Hypermethylation: Epigenetic mechanisms

Since the 1980s, studies that have examined the effects of maternal nutrition during pregnancy on the risk of degenerative diseases later in life have indicated that fetal development can be affected by maternal over- and undernourishment ${ }^{(1-5)}$. The most famous researchers in this field are Barker et al. (1), who studied the association between the mortality rates of CVD and neonatal mortality more than 70 years ago in different areas of England and Wales. They suggested that poor nutrition in early life increased susceptibility to CVD ${ }^{(2)}$. Population cohort studies have indicated that adults who were exposed to the Dutch famine (which occurred during the winter of 1944-5) in utero tended to have an atherogenic lipid profile ${ }^{(6)}$, high BMI, large waist circumference ${ }^{(7)}$ and high risk of $\mathrm{CHD}^{(8)}$. These studies gave evidence that showed an association between nutritional experience in early life and the risk of degenerative disease in later life. They also gave clear evidence that degenerative diseases, such as CHD, hypertension, dyslipidaemia, obesity and diabetes, could be initiated in utero.

Several animal models have been developed to investigate the molecular mechanism. Researchers have designed several diets, including energy-rich diets $^{(9)}$, protein-restrictive diets ${ }^{(10)}$ and micronutrient-deficient diets ${ }^{(11)}$, to feed pregnant and/or lactational rodents in order to study the effects of maternal diet on the health status of offspring in later life. Some studies $^{(12,13)}$ have shown metabolic disorder in the offspring of dams that were fed a high-lipid, high-energy (HLE) diet during gestation and lactation; however, the specific molecular mechanisms underlying these changes were not very clear. Currently, the most famous mechanism is epigenetics ${ }^{(14)}$. In 1998, Wolff et al. ${ }^{(15)}$ reported that feeding pregnant black a/a dams methyl-supplemented diets (supplemented with folic acid, vitamin $\mathrm{B}_{12}$, betaine and choline) changed the epigenetic regulation of agouti expression in their offspring. That study indicated that the maternal diet is important for the epigenetics of offspring. Some researchers have tested this hypothesis and have found that altering the maternal diet, e.g. by introducing a protein-deficient diet ${ }^{(10,16)}$ or a micronutrient-deficient $\operatorname{diet}^{(15)}$, changed the DNA methylation profile in offspring. Recently, Cannon et $a l .{ }^{(17)}$ reported that maternal diet has a pervasive effect on gene expression, but transcriptional changes are unlikely to be caused by DNA methylation differences. However, Marco et al. ${ }^{(18)}$ reported that even when offspring from dams that were fed HLE diets during gestation and lactation

Abbreviations: CD, chow diet; GO, gene ontology; HLE, high-lipid, high-energy; $L X R \alpha$, liver X receptor $\alpha$.

* Corresponding author: R. Xiao, email xiaor22@ccmu.edu.cn

†These authors contributed equally to the present work. 
ate chow diet (CD) from weaning, the offspring displayed increased Pomc promoter hypermethylation. This discrimination was limited by sample size, body weight before gestation, diet composition and other factors.

In our earlier study ${ }^{(19)}$, we reported that global gene expression decreased in neonatal mice livers in order to adapt to a maternal HLE diet during gestation and lactation. It has been suggested that the maternal consumption of a high-fat diet can change the epigenetic marks in offspring and alter gene expression in the long term ${ }^{(20)}$. In the present study, we used microarrays to analyse the global methylation changes in gene promoter sites in order to find the mechanism that underlies the global decreasing gene expression that we observed in our earlier study.

\section{Material and method}

\section{Animals and diets}

The Beijing Administrative Committee for Laboratory Animals and the Ethical Committee for Animal Care and Use of the Capital Medical University reviewed and approved all animal experiments carried out in the present study.

Male and female C57BL/6J mice (specific pathogen free, 9 weeks old) were housed in groups of four mice per cage at $22^{\circ} \mathrm{C}$ with a $12 \mathrm{~h}$ light $-12 \mathrm{~h}$ dark cycle, and they were given free access to food and water. After 1 week, fifteen male and fifteen female mice were transferred to one cage and fed either a control CD or an HLE diet (composed of $84 \% \mathrm{CD}+15 \cdot 8 \%$ lard fat $+0.2 \%$ cholesterol). These same diets were used in one of our previous studies ${ }^{(17)}$. Total food intake and body weight gain were recorded twice a week. There were ten pregnant CD dams and ten pregnant HLE dams that gave birth to litters ranging from six to ten pups. One male pup from each dam was randomly assigned to the present experiment. After $21 \mathrm{~d}$, all of the weaning offspring ( $n 10$ in each group) were fed the CD until they were 10 weeks old, at which time they were killed by cervical vertebra dislocation. Livers were sampled, immediately frozen in liquid $\mathrm{N}_{2}$ and stored at $-80^{\circ} \mathrm{C}$ until further study.

\section{Genomic DNA, total RNA, and protein isolation from livers}

Total RNA, genomic DNA and proteins were purified by commercial kits (GenElute RNA/DNA/Protein Plus Purification Kit, catalogue number E5163) provided by Sigma. All of the procedures were conducted according to the manufacturer's instructions. In brief, $20 \mathrm{mg}$ liver tissues from one of the twenty mice were minced thoroughly using a pestle and transferred into a DNA purification column. After spinning and washing repeatedly, the genomic DNA, total RNA and protein were separated and purified.

\section{Global DNA methylation quantification}

The methylation of the genomic DNA was detected using the Methylamp global DNA methylation quantification ultra kit
(Epigentek) according to the manufacturer's operational guide. In this assay, genomic DNA is immobilised in strip wells specifically coated with a DNA-affinity substance. The methylated fraction of DNA can be recognised by a 5-methylcytosine antibody and quantified through an ELISA-like reaction. The amount of methylated DNA is proportional to the optical density (OD) intensity. Absorbance of positive (methylated) and negative (unmethylated) control DNA were measured by a microplate reader (Tecan). The amount of DNA methylation was calculated using the following formula:

\section{Methylation \%}

$$
=\frac{(\text { sample OD }- \text { negative control }) / X^{*}}{(\text { positive control OD }- \text { negative control OD }) \times 10} \times 100 \% \text {. }
$$

The amount of the positive control was $10 \mathrm{ng}$, and sample DNA was adjusted to $100 \mathrm{ng} . X^{*}$ is the GC content of the species DNA; in the present study, it is $42 \%$.

\section{Microarray analysis of DNA methylation and data analysis}

The Mouse Promoter 1.0R Array (Affymetrix) was used to detect DNA methylation. It is a single array comprised of more than 4.6 million probes that are tiled to interrogate more than 28000 mouse promoter regions. The entire procedure was conducted according to the manufacturer's instructions. In brief, genomic DNA was purified using a kit (Qiagen) on three samples from each group. Each sample included a mixture of DNA from three mice livers that were randomly selected from the ten livers. Methylated DNA was gathered using the EpiQuik Methylated DNA Immunoprecipitation Kit (Epigentek). Target DNA was amplified, fragmented and labelled. After hybridisation, washing and staining, the chip was scanned according to the manufacturer's instructions. First, all of the locations where the degree of methylation was significantly changed were identified, and the gene names were annotated. We applied a random variance model (RVM) $t$ test to filter the differentially expressed methylation peak genes for the control and experiment groups, because an RVM $t$ test can raise df effectively in the case of small samples. After the significant analysis and false discovery rate analysis, we selected the differentially expressed genes according to the $P$ value threshold ${ }^{(21-23)}$.

Then, all of the genes that had been identified by microarrays as being significantly hypermethylated were analysed using gene ontology (GO) analysis and pathway analysis, which were outlined in a study that we published earlier ${ }^{(19)}$.

\section{Gene expression analysis by real-time PCR}

The total RNA that had been purified earlier was reverse transcribed using an RT kit (no. A3500; Applied Promega). The mRNA encoding PPAR $\gamma$, liver X receptor $\alpha(L X R \alpha)$, cluster of differentiation 36 (CD36) and $\beta$-actin (which was used as an invariant control) were analysed by real-time PCR. The primer sequences are listed in Table 1. PCR was carried out 
Table 1. Primers sequence used in the present study

\begin{tabular}{llc}
\hline & Sequence $\left(5^{\prime}-3^{\prime}\right)$ & Product $(\mathrm{bp})$ \\
\hline$\beta$-Actin & F: TGGAATCCTGTGGCATCCATGAAAC & 348 \\
& R: TAAAACGCAGCTCAGTAACAGTCCG & \\
$P P A R \gamma$ & F: GTGCTCCAGAAGATGACAGAC & 158 \\
& R: GGTGGGACTTTCCTGCTAATAC & 134 \\
$L X R \alpha$ & F: TGTGGAAGACAGAACCTCAAGA & 134 \\
& R: TATCCCTAAAGCAACCCCAGTT & 183 \\
CD36 & F: CGTCTGCTCACAGCCTCATA & \\
& R: CATCGTTTCCCACACTCCTT & \\
\hline
\end{tabular}

F, forward; R, reverse; $L X R \alpha$, liver $X$ receptor $\alpha ; C D 36$, cluster of differentiation.

as follows: denaturation at $94^{\circ} \mathrm{C}$ for $5 \mathrm{~min}$ for the first cycle, and then cycles consisting of denaturation at $94^{\circ} \mathrm{C}$ for $30 \mathrm{~s}$, annealing at $60^{\circ} \mathrm{C}$ for $30 \mathrm{~s}$ and extension at $72^{\circ} \mathrm{C}$ for $30 \mathrm{~s}$.

\section{Western blot analysis}

For the Western blot analysis, livers from adult mice were homogenised on ice in radioimmunoprecipitation assay (RIPA) lysis buffer (Sigma) containing phenylmethanesulfonyl fluoride as a proteinase inhibitor. Homogenates were centrifuged for $10 \mathrm{~min}$ at $12000 \mathrm{rpm}$ at $4^{\circ} \mathrm{C}$, and the supernatants were collected. The total protein content was determined using the bicinchoninic acid method. Then, $50 \mu \mathrm{g}$ of protein extracts from each liver tissue were resuspended in an SDS-containing buffer, maintained in boiling water for $5 \mathrm{~min}$ and separated by SDS-PAGE. After electrophoresis, proteins were transferred to nitrocellulose membranes, and PPAR $\gamma$ and $L X R \alpha$ were detected using an anti-PPAR $\gamma$ antibody (ab3259, 1:1000) and an anti-LXR $\alpha$ antibody (ab58092, 1:1000). An anti- $\beta$-actin antibody (ab, 1:1000) was used as a control. Signals were revealed using an enhanced chemiluminescence (ECL) kit (Fivephoton Biochemicals), and the dilution ratio of IgG was 1:5000. ImageJ was used to analyse the density of the bands.

\section{Statistical analysis}

Results are expressed as means with their standard errors. SPSS (version 11.5; SPSS Institute, Inc.) was used for statistical analysis. Significant differences were assessed using Student's $t$ test (two-tailed). A $P$ value of $<0.05$ was considered statistically significant.

\section{Results}

The genomic DNA in adult mice livers was hypermethylated by a maternal high-lipid, high-energy diet during pregnancy and lactation

The global DNA methylation degree was measured using commercial kits. The results indicated that the percentage of methylated DNA in mice livers from offspring delivered by dams that were fed an HLE diet during pregnancy and lactation was significantly higher than that in the CD group (Fig. 1). The percentage of methylated DNA in the CD group ( $n$ 10) was $24.55 \%$, whereas it was $29 \cdot 24 \%$ in the HLE group ( $n$ 10).
The hypermethylated genes in adult mice livers were identified by microarray

There were 1635 genes identified as being significantly hypermethylated by a maternal HLE diet (online supplementary Table S1), including some famous genes related to glucose, fatty acids and cholesterol metabolism, such as $P P A R \gamma$, nuclear receptor subfamily 1 , group $\mathrm{H}$, member 3 (NR1H3) (LXR $\alpha)$, Insig2 (insulin induced gene 2), CD36 and Pcsk9 (proprotein convertase subtilisin/kexin type 9) (refer to online supplementary Table S1). All of these hypermethylated genes were distributed in the nineteen chromosomes shown in Fig. 2.

\section{Bioinformatic analysis mapped the relationship between} the functions of the genes which were hypermethylated

Then, all of the genes that had been identified as being significantly hypermethylated were analysed using GO analysis and pathway analysis, which were described in a study that we published earlier ${ }^{(19)}$. GO analysis indicated that there were $261 \mathrm{GO}$ (online supplementary Table S2) significantly affected by the maternal diet. GO map analysis helped determine the relationship between the differentially expressed and upstream genes. In this map, a total of twenty-four groups of functions were affected, including phosphorylation protein (GO:0006468), the Wnt receptor signalling pathway (GO:0016055), the regulation of the apoptotic process (GO:0042981) and the apoptotic process (GO:0006915) etc. (Fig. 3).

Functions related to lipid storage (GO:0019915), the lipid metabolic process (GO:0006629), the negative regulation of LDL particle clearance (GO:0010989), cholesterol homeostasis (GO:0042632), the fatty acid biosynthetic process (GO:0006633), the long-chain fatty acid metabolic process (GO:0001676), the sphingolipid metabolic process (GO:0006665), the regulation of the steroid metabolic process (GO:0019218), the regulation of cholesterol transport (GO:0032374), the regulation of the LDL particle receptor catabolic process (GO:0032803), the long-chain fatty-acyl-CoA metabolic process (GO:0035336), multicellular organismal lipid catabolism (GO:0044240), LDL particle

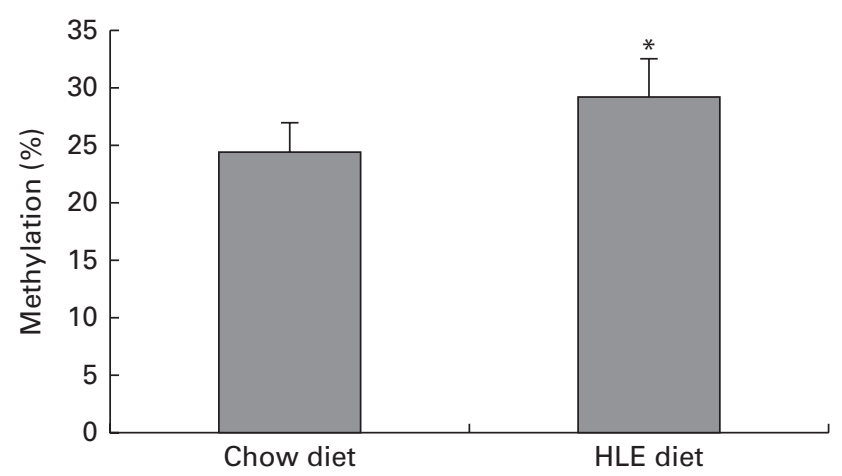

Fig. 1. The amount of methylated DNA in the livers of adult male offspring from dams that were fed a chow diet (CD) or a high-lipid, high-energy (HLE) diet during pregnancy and lactation. Genomic DNA from mice liver was extracted, and the methylated DNA was captured by a 5-methylcytosine antibody and quantified through an ELISA-like reaction. Values are means ( $n 10)$, with their standard errors represented by vertical bars. ${ }^{*}$ Mean value was significantly different compared to that in the CD group $(P \leq 0.05)$. 
$\log _{2}$ (ratio)

Chromosome graph

I

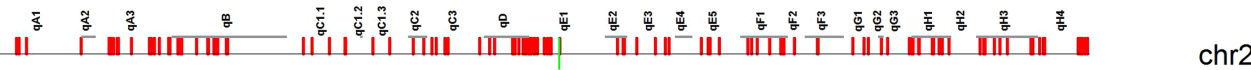

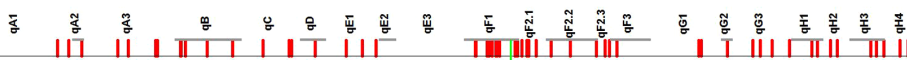

chr3

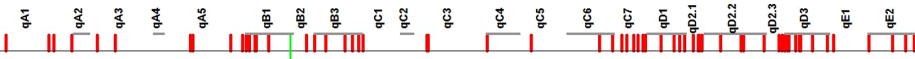

chr4

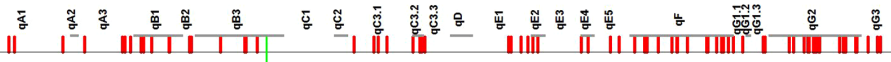

chr5

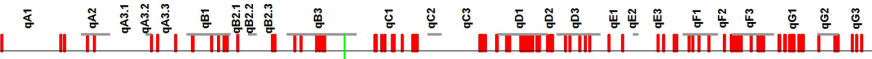

chr6

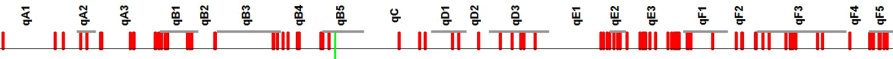

chr7

|

chr8

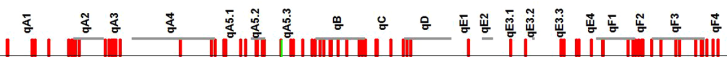

chr9

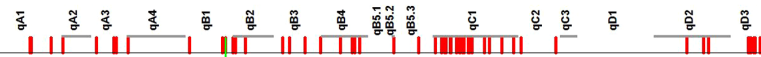

chr10

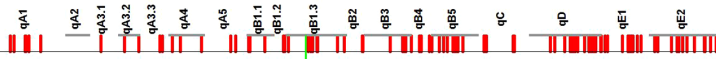

|

chr11

chr12

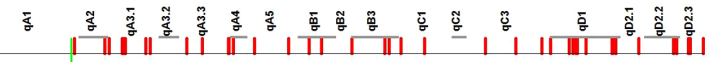

chr13

ป

chr14

I

chr15

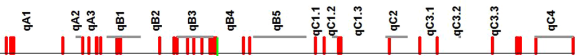

chr16

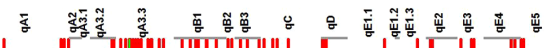

chr17

II।

chr18

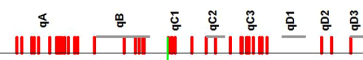

chr19

Fig. 2. The localisation of hypermethylated genes in chromosomes (chr) recognised by the mouse promoter 1.0R Array provided by Affymetrix. $\square$, Hypermethylation; $\square$, centromere. A colour version of this figure can be found online at http://www.journals.cambridge.org/bjn

mediated signalling (GO:0055096), the response to LDL particle stimulus (GO:0055098), the lipid biosynthetic process (GO:0008610), the TAG metabolic process (GO:0006641), the saturated monocarboxylic acid metabolic process (GO:0032788), the unsaturated monocarboxylic acid metabolic process (GO:0032789) and lipid transport (GO:0006869) were also significantly affected.

Pathway analysis found that there were fifty-five pathways (online supplementary Table S3) that were affected by the maternal diet, including metabolic pathways, pathways in cancer, the mitogen-activated protein kinase signalling pathway, the Wnt signalling pathway and the Ca signalling pathway etc. The insulin signalling pathway (pathway ID: 4910), the biosynthesis of unsaturated fatty acid pathway (pathway ID: 1040) and the PPAR signalling pathway (pathway ID: 3320) were also significantly affected by the maternal diet. Some of these pathways interacted with each other, as was revealed by the pathway net analysis based on the Kyoto Encyclopedia of Genes and Genomes (KEGG) database (Fig. 4). The analysis indicated that the insulin signalling pathway was an upstream pathway, and changes to it resulted in changes in other pathways. 


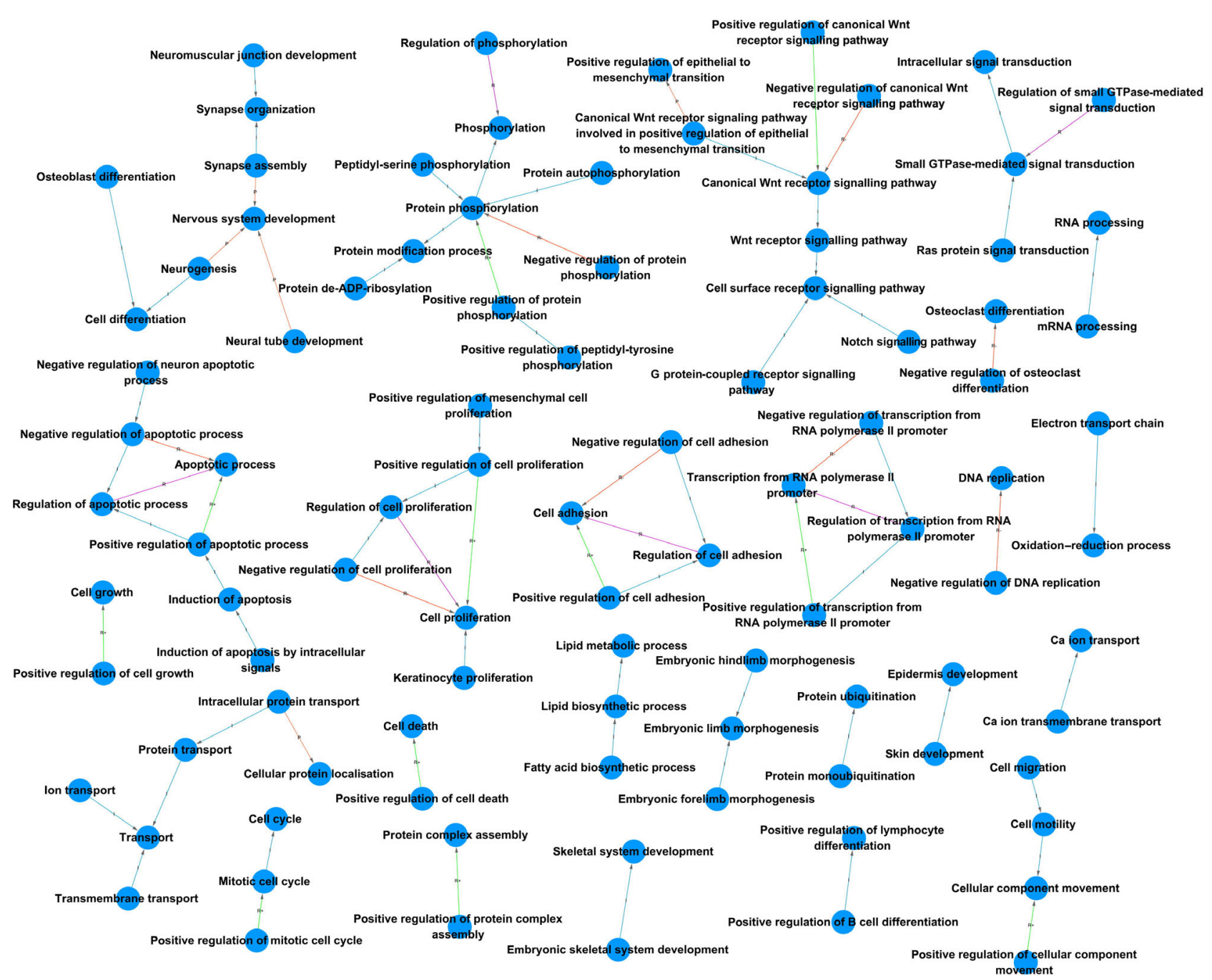

Fig. 3. Gene ontology (GO) map made from significant GO regulated by a maternal high-lipid, high-energy (HLE) diet. Based on the GO analysis, significant GO affected by a maternal HLE diet during gestation and lactation were selected, and the GO map was made. All of these GO were down-regulated. A colour version of this figure can be found online at http://www.journals.cambridge.org/bjn

The expression of genes related to lipid metabolism were inhibited by a maternal high-lipid, high-energy diet as measured by real-time PCR and Western blot

The PPAR,$L X R \alpha$ and $C D 36$ genes, which are related to lipid metabolism in hepatic tissues of adult offspring, were found by microarray to be hypermethylated. DNA methylation leads to the inhibition of gene transcription. The mRNA and protein expression of these genes were therefore measured by real-time PCR and Western blot analysis. The results indicated that PPAR $\gamma$ and $L X R \alpha$ mRNA and protein expression were significantly decreased in the HLE group (Fig. 5(a) and (b)). The CD36 mRNA expression was also significantly inhibited by a maternal HLE diet (Fig. 5(a)).

\section{Discussion}

It has been reported that a maternal HLE diet during pregnancy induces the global inhibition of gene expression in neonatal mice livers, and lower levels of the expression of these genes remained in adulthood when offspring were fed $\mathrm{CD}$ after weaning ${ }^{(19)}$. We hypothesised that there might be global gene promoter hypermethylation in the liver. Using the Mouse Promoter 1.0R Array, we carried out a genomewide analysis of the DNA methylation of gene promoters in adult mouse livers to verify this hypothesis. The results indicated that the promoter hypermethylation of genes existed, and some of these genes were involved in the lipid metabolism and pathways.

Intra-uterine life is a complex developmental programme that involves cell division, growth and differentiation ${ }^{(24)}$. The ability to sense, interpret and react to environmental stimuli by changing gene expression is a basic survival skill of cells. This process includes the modification of DNA by methylation at $\mathrm{CpG}$ sites ${ }^{(25)}$. Nutrients transferred from dams to fetuses are important environmental stimuli. It has been reported that a maternal low-protein diet significantly increases the methylation of $P P A R \alpha$ in offspring livers ${ }^{(26)}$. In another study, global and gene-specific (dopamine reuptake transporter, $\mu$-opioid receptor and preproenkephalin) promoter DNA 


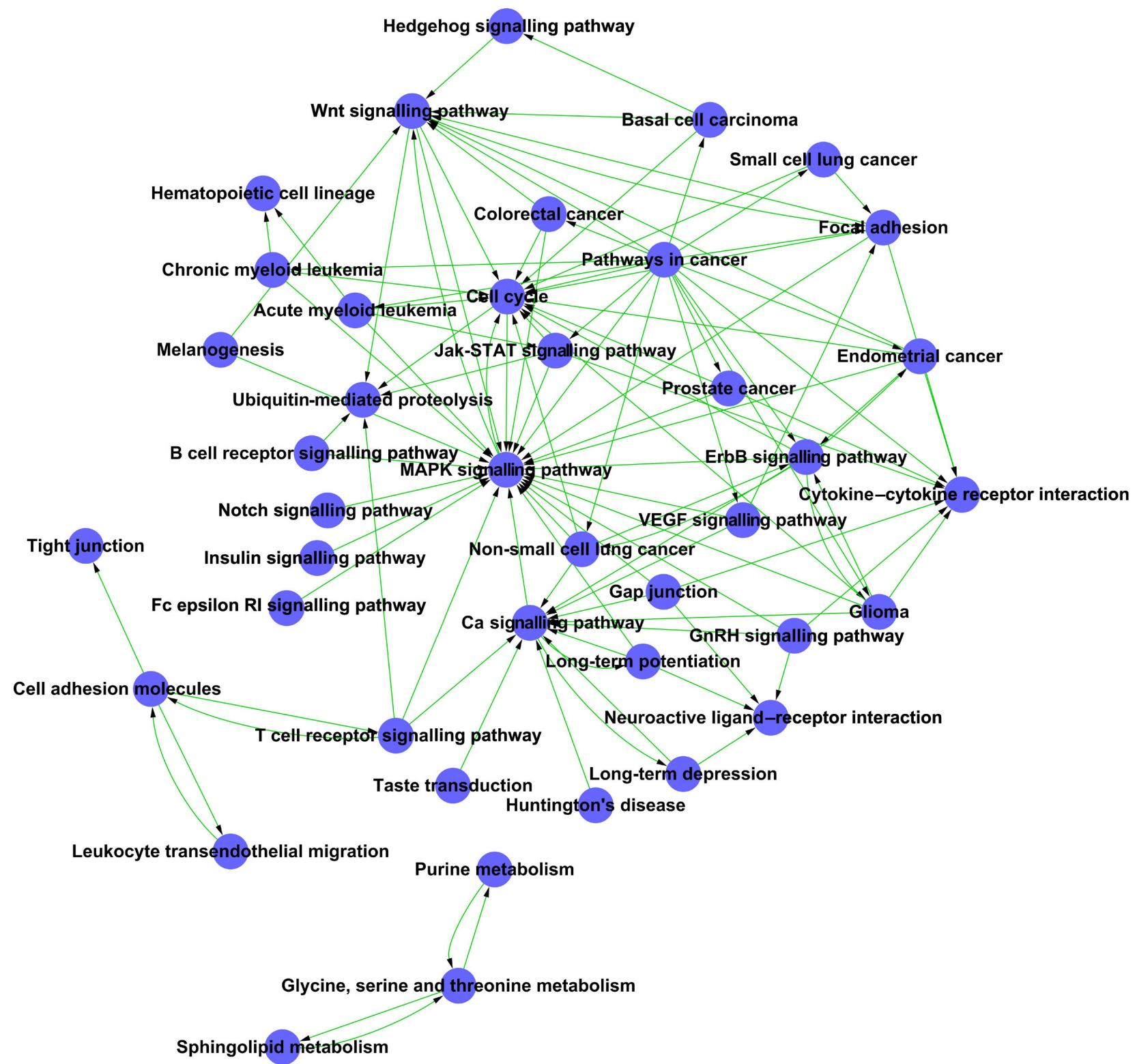

Fig. 4. Pathway net constructed from significant pathways regulated by a maternal high-lipid, high-energy (HLE) diet during gestation and lactation. Based on the Kyoto Encyclopedia of Genes and Genomes (KEGG) database, fifty-five significantly changed pathways were identified, and a pathway network was created. MAPK, mitogen-activated protein kinase. A colour version of this figure can be found online at http://www.journals.cambridge.org/bjn

hypomethylation were observed in the brains of offspring from dams that were fed a high-fat diet ${ }^{(18)}$. In oocytes of obese mice that were induced by a high-fat diet, the leptin promoter DNA was significantly hypermethylated, but the $\operatorname{PPAR} \alpha$ was hypomethylated ${ }^{(27)}$. This trend was also observed in the livers of female offspring from dams that were fed a high-fat diet ${ }^{(27)}$. These results indicated that the epigenetic mechanism underlies the effects of maternal diets on gene expression in offspring. However, there is significant discrepancy. Recently, a study reported that a maternal high-fat diet had a pervasive effect on gene expression but not on DNA methylation in the liver ${ }^{(17)}$, but another research group reported that offspring from dams that were fed an HLE diet during gestation and lactation displayed increased hypermethylation at the promoter of proopiomelanocortin gene ${ }^{(18)}$.
In the present study, the specific genes whose promoter DNA were significantly hypermethylated or hypomethylated were scanned using microarray on liver tissue. Among the 1635 genes, only seventeen genes were hypomethylated significantly, while the others were significantly hypermethylated in the livers of offspring from dams that were fed an HLE diet. Because of the absolute advantage of the hypermethylation of genes, the results of the global DNA methylation measurements indicated that the percentage of methylated DNA in livers in the HLE group was significantly higher than that in the CD group. The significant heterogeneity of factors such as the fatty acids category in the diet, the genes that were selected to be observed and the number of dams and offspring used in the studies ${ }^{(17,28)}$ made inconsistent results between the studies predictable. 


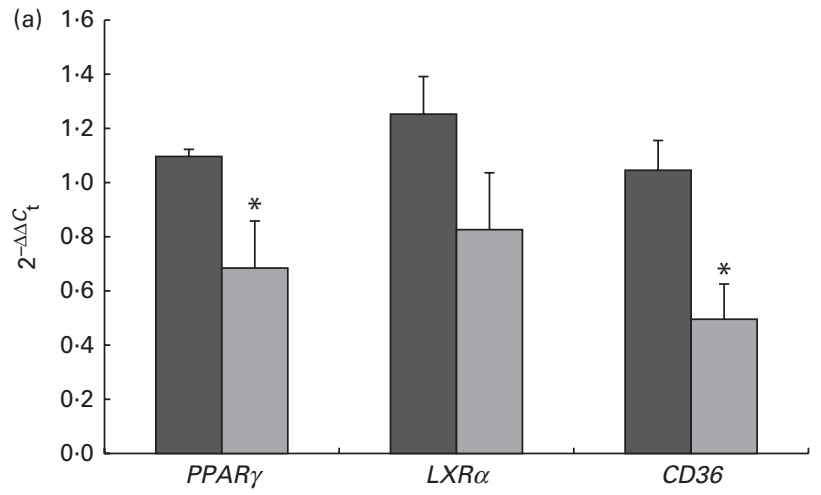

(b)
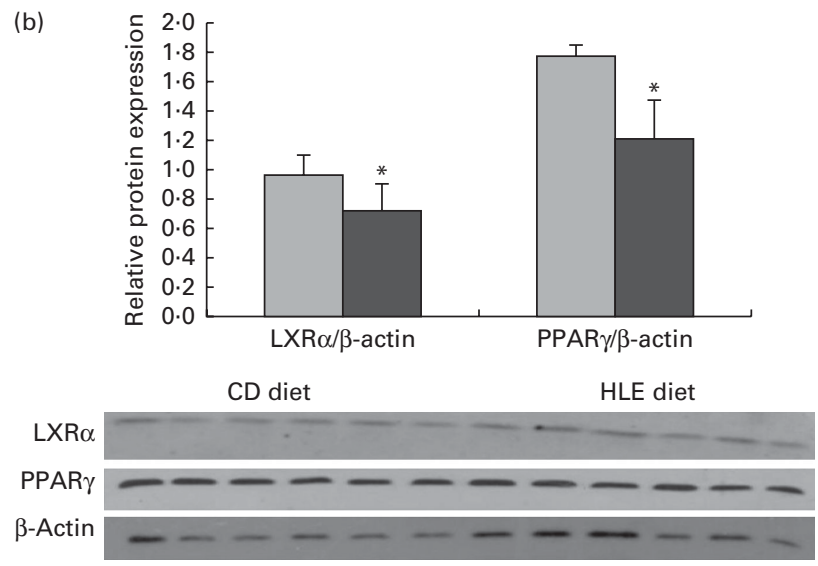

Fig. 5. Expression levels of mRNA and protein in livers of male adult offspring from dams fed a chow diet (CD) or high-lipid, high-energy (HLE) diet. (a) The mRNA levels of PPAR,$L X R \alpha$ (liver $\mathrm{X}$ receptor $\alpha$ ) and $C D 36$ (cluster of differentiation 36 ) in male adult mice livers measured by real-time PCR ( $n$ 7). $\square, \mathrm{CD} ; \square$, HLE diet. (b) The nuclear PPAR $\gamma$ and LXR $\alpha$ protein content in male adult mice livers measured by Western blot $(n 6)$. $\square$, CD; $\square$, HLE diet. Values are means, with their standard errors represented by vertical bars. * Mean value was significantly different compared to $C D(P \leq 0.05)$.

Many studies found down-regulation in some important genes that regulate lipid and glucose metabolism. In Laker et al.'s study ${ }^{(29)}$, the mRNA expression of PPAR $\gamma$ co-activator$1 \alpha(P g c-1 \alpha)$ and its target genes were inhibited in the muscular tissue of neonatal and adult mice from dams that were fed a high-fat diet during gestation and lactation. Magliano et al. ${ }^{(30)}$ found that a maternal pre-gestational and gestational high-fat diet diminished the expression of PPAR $\alpha$ in the livers and PPAR $\gamma$ in the white adipose tissue of adult offspring. In the present study, the nuclear protein levels of PPAR $\gamma$ and $L X R \alpha$ were significantly decreased in the livers of mice that were the offspring of dams that were fed an HLE diet. Both PPAR $\gamma$ and $L X R \alpha$ were also suggested as hypermethylated genes by microarray measurement.

$P P A R \gamma$ was found predominantly in fat tissues and to a lesser extent in other tissues, including the liver ${ }^{(31)}$. $P P A R \gamma$ plays multiple roles in regulating cell functions, including fatty acid storage and glucose metabolism. The genes regulated by $P P A R \gamma$ stimulate lipid uptake and adipogenesis in adipocytes. In livers, $P P A R \gamma$ participated in the regulation of steatosis, which was induced by a high-fat diet in mice ${ }^{(32)}$. In Ashino et al.'s study ${ }^{(33)}$, the expression of fatty acid synthase (FAS) and acetyl-CoA carboxylase (ACC) in the liver was significantly lower in adult offspring from dams that were fed a high-fat diet than that in the CD group, which corroborates our earlier finding that fatty acid synthesis was not stimulated by a maternal high-lipid diet in adult mice that were fed CD after weaning ${ }^{(19)}$. In the present study, global methylation in gene promoter sites might explain the low expression level of genes.

$L X R \alpha$ is another nuclear receptor that regulates the metabolism of several important lipids. The predominant function of $L X R \alpha$ is to regulate intracellular cholesterol balance by inducing the gene expression of cholesterol $7 \alpha$-hydroxylase and ATP-binding cassette transporters ${ }^{(34)}$. In a rabbit model, the low expression of $L X R \alpha$ in the placenta was found in male offspring of dams that were fed high-lipid diets ${ }^{(35)}$. It has been reported that promoter sites were hypermethylated in mice offspring from dams that were fed protein-restricted diets during gestation ${ }^{(36)}$. In the present study, the expression of liver $L X R \alpha$ was inhibited by a maternal HLE diet, and hypermethylation at promoter sites might be involved.

In the present study, GO related to cell proliferation, protein phosphorylation, cell adhesion, apoptotic process and DNA replication were affected. Pathways related to cancer were significantly affected. The fetal developmental process is also a process of cell differentiation and proliferation ${ }^{(19)}$. The present study indicated the possibility that most cancer-related pathways were affected, and this hypothesis needs to be verified in the future.

In conclusion, the present study indicates that a maternal high-fat diet during gestation and lactation induced global DNA hypermethylation, including fatty acid and cholesterol metabolism-related genes.

\section{Supplementary material}

To view supplementary material for theis present article, please visit http://dx.doi.org/10.1017/S0007114515000252

\section{Acknowledgements}

The authors would like to acknowledge the technical assistance of the Genminix Company (Shanghai, China).

The present work was funded by grants from the Nature Science Foundation of China (81273070, 81102122 and 30901197).

The authors declared that there are no competing financial interests in relation to the present work. The authors state that there is no conflict of interest.

H.-L. Y. and R. X. conceived of and designed the experiments. S. D., L.-F. G., L. L., Y.-D. Xi. and W.-W. M. performed the experiments. S. D. and L.-H. Y. analysed the data. H.-L. Y. wrote the paper.

\section{References}

1. Barker DJ, Osmond C \& Law CM (1989) The intrauterine and early postnatal origins of cardiovascular disease and chronic bronchitis. J Epidemiol Community Health 43, 237-240. 
2. Barker DJ (1991) The intrauterine environment and adult cardiovascular disease. Ciba Found Symp 156, 3-10.

3. Li Y, Jaddoe VW, Qi L, et al. (2011) Exposure to the Chinese famine in early life and the risk of hypertension in adulthood. J Hypertens 29, 1085-1092.

4. McMillen IC, MacLaughlin SM, Muhlhausler BS, et al. (2008) Developmental origins of adult health and disease: the role of periconceptional and foetal nutrition. Basic Clin Pharmacol Toxicol 102, 82-89.

5. Vickers MH, Breier BH, Cutfield WS, et al. (2000) Fetal origins of hyperphagia, obesity, and hypertension and postnatal amplification by hypercaloric nutrition. Am J Physiol Endocrinol Metab 279, E83-E87.

6. Roseboom TJ, van der Meulen JH, Osmond C, et al. (2000) Plasma lipid profiles in adults after prenatal exposure to the Dutch famine. Am J Clin Nutr 72, 1101-1106.

7. Ravelli AC, van Der Meulen JH, Osmond C, et al. (1999) Obesity at the age of $50 \mathrm{y}$ in men and women exposed to famine prenatally. Am J Clin Nutr 70, 811-816.

8. Roseboom TJ, van der Meulen JH, Osmond C, et al. (2000) Coronary heart disease after prenatal exposure to the Dutch famine, 1944-45. Heart 84, 595-598.

9. Williams L, Seki Y, Vuguin PM, et al. (2014) Animal models of in utero exposure to a high fat diet: a review. Biochim Biophys Acta 1842, 507-519.

10. Altobelli G, Bogdarina IG, Stupka E, et al. (2013) Genomewide methylation and gene expression changes in newborn rats following maternal protein restriction and reversal by folic acid. PLOS ONE 8, e82989.

11. Tomat AL, Costa Mde L \& Arranz CT (2011) Zinc restriction during different periods of life: influence in renal and cardiovascular diseases. Nutrition 27, 392-398.

12. Melo AM, Benatti RO, Ignacio-Souza LM, et al. (2014) Hypothalamic endoplasmic reticulum stress and insulin resistance in offspring of mice dams fed high-fat diet during pregnancy and lactation. Metabolism 63, 682-692.

13. Benatti RO, Melo AM, Borges FO, et al. (2014) Maternal high-fat diet consumption modulates hepatic lipid metabolism and microRNA-122 (miR-122) and microRNA-370 (miR-370) expression in offspring. Br J Nutr 111, $2112-2122$

14. Osborne-Majnik A, Fu Q \& Lane RH (2013) Epigenetic mechanisms in fetal origins of health and disease. Clin Obstet Gynecol 56, 622-632.

15. Wolff GL, Kodell RL, Moore SR, et al. (1998) Maternal epigenetics and methyl supplements affect agouti gene expression in Avy/a mice. FASEB J 12, 949-957.

16. Rees WD, Hay SM, Brown DS, et al. (2000) Maternal protein deficiency causes hypermethylation of DNA in the livers of rat fetuses. J Nutr 130, 1821-1826.

17. Cannon MV, Buchner DA, Hester J, et al. (2014) Maternal nutrition induces pervasive gene expression changes but no detectable DNA methylation differences in the liver of adult offspring. PLOS ONE 9, e90335.

18. Marco A, Kisliouk T, Tabachnik T, et al. (2014) Overweight and $\mathrm{CpG}$ methylation of the Pomc promoter in offspring of high-fat-diet-fed dams are not "reprogrammed" by regular chow diet in rats. FASEB J (Epublication ahead of print version 13 June 2014).

19. Yu HL, Miao HT, Gao LF, et al. (2013) Adaptive responses by mouse fetus to a maternal HLE diet by downregulating
SREBP1: a microarray- and bio-analytic-based study. I Lipid Res 54, 3269-3280

20. Vucetic Z, Kimmel J, Totoki K, et al. (2010) Maternal high-fat diet alters methylation and gene expression of dopamine and opioid-related genes. Endocrinology 151, 4756-4764.

21. Wright GW \& Simon RM (2003) A random variance model for detection of differential gene expression in small microarray experiments. Bioinformatics 19, 2448-2455.

22. Yang H, Crawford N, Lukes L, et al. (2005) Metastasis predictive signature profiles pre-exist in normal tissues. Clin Exp Metastasis 22, 593-603.

23. Clarke R, Ressom HW, Wang A, et al. (2008) The properties of high-dimensional data spaces: implications for exploring gene and protein expression data. Nat Rev Cancer 8, 37-49.

24. Bird A (2007) Perceptions of epigenetics. Nature $\mathbf{4 4 7}$, 396-398.

25. Jaenisch R \& Bird A (2003) Epigenetic regulation of gene expression: how the genome integrates intrinsic and environmental signals. Nat Genet 33, S245-S254.

26. Altmann S, Murani E, Schwerin M, et al. (2013) Dietary protein restriction and excess of pregnant German Landrace sows induce changes in hepatic gene expression and promoter methylation of key metabolic genes in the offspring. J Nutr Biochem 24, 484-495.

27. Ge ZJ, Luo SM, Lin F, et al. (2014) DNA methylation in oocytes and liver of female mice and their offspring: effects of high-fat-diet-induced obesity. Environ Health Perspect 122, 159-164

28. Llopis M, Sánchez J, Priego T, et al. (2014) Maternal fat supplementation during late pregnancy and lactation influences the development of hepatic steatosis in offspring depending on the fat source. J Agric Food Chem 62, 1590-1601.

29. Laker RC, Lillard TS, Okutsu M, et al. (2014) Exercise prevents maternal high-fat diet-induced hypermethylation of the $P g c-1 \alpha$ gene and age-dependent metabolic dysfunction in the offspring. Diabetes $\mathbf{6 3}$, e6-e7.

30. Magliano DC, Bargut TC, de Carvalho SN, et al. (2013) Peroxisome proliferator-activated receptors- $\alpha$ and $\gamma$ are targets to treat offspring from maternal diet-induced obesity in mice. PLOS ONE 8, e64258.

31. Tsai YS \& Maeda N (2005) PPAR $\gamma$ : a critical determinant of body fat distribution in humans and mice. Trends Cardiovasc Med 15, 81-85.

32. Inoue $\mathrm{M}$, Ohtake $\mathrm{T}$, Motomura $\mathrm{W}$, et al. (2005) Increased expression of PPAR $y$ in high fat diet-induced liver steatosis in mice. Biochem Biophys Res Commun 336, 215-222.

33. Ashino NG, Saito KN, Souza FD, et al. (2012) Maternal highfat feeding through pregnancy and lactation predisposes mouse offspring to molecular insulin resistance and fatty liver. J Nutr Biochem 23, 341-348.

34. Zhang Y, Chan JF \& Cummins CL (2009) Liver X receptors as therapeutic targets for managing cholesterol: implications for atherosclerosis and other inflammatory conditions. Clin Lipidol 4, 29-40.

35. Tarrade A, Rousseau-Ralliard D, Aubrière MC, et al. (2013) Sexual dimorphism of the feto-placental phenotype in response to a high fat and control maternal diets in a rabbit model. PLOS ONE 8, e83458.

36. van Straten EM, Bloks VW, Huijkman NC, et al. (2010) The liver X-receptor gene promoter is hypermethylated in a mouse model of prenatal protein restriction. Am J Physiol Regul Integr Comp Physiol 298, R275-R282. 